\title{
Properties of Indium Tin Oxide Thin Films Deposited on Polymer Substrates
}

\author{
S.H. Mohamed ${ }^{a}$, F.M. El-Hossary ${ }^{a}$, G.A. GAmal ${ }^{b}$ And M.M. KAhlid ${ }^{b}$ \\ ${ }^{a}$ Physics Department, Faculty of Science, Sohag University, 82524 Sohag, Egypt \\ ${ }^{b}$ Physics Department, Faculty of Science, South Valley University \\ 83523 Qena, Egypt
}

(Received September 5, 2008; revised version October 21, 2008)

\begin{abstract}
Indium tin oxide thin films with different thicknesses were deposited on polymer substrates, held at room temperature, using electron beam evaporation. The dependence of structural properties, optical properties and room temperature resistivity on the indium tin oxide film thickness was studied. X-ray diffraction illustrates the amorphous structure for all the indium tin oxide prepared films. The high roughness of the polymer substrate affects the properties of indium tin oxide films. The transmittance, the resistivity, and the optical band gap decrease with increasing the film thickness while the refractive index increases. The present indium tin oxide films are amorphous, transparent and have relatively low resistivity. These properties are suitable as transparent electrode for organic light-emitting diodes, touch screens, and in piezoelectric applications.
\end{abstract}

PACS numbers: 61.05.cp, 07.79.Lh, 78.66.--w, 73.61.-r

\section{Introduction}

Indium tin oxide (ITO) thin films are widely used in optoelectronic devices such as solar cells, electroluminescence and liquid crystal displays as transparent electrode due to its high transmittance in the visible region and low electrical resistivity [1-3]. The high electrical conductivity of ITO films results in a high reflectivity in the infrared region, which gives applications to thermal insulation of windows, prevention of radiative cooling [4], etc.

Recently, there has been increasing interest in depositing ITO film on polymer substrates for light-emitting diode (LED) [5], organic light-emitting devices (OLED) [6], and liquid crystal display (LCD) applications [7]. Compared with the traditional glass substrate, polymer substrates provide displays that are lighter in weight and more resistant to impact damage, making them suitable for portable devices. An LCD or OLED built on flexible materials, such as metal and plastic, can be made into any shape, making it ideal for a car dashboard or a handy roll-up. Though the technical hurdles are significant, flexible displays could lead to major gains in manufacturing efficiency. Instead of relying on batch processes used for LCDs, the displays could be made on roll-to-roll continuous-processing equipment, much like the printers are used to print newspapers [8].

Amorphous transparent conductive (TC) films are currently important for the usage as a transparent electrode of organic light-emitting diode, because of their surface flatness and availability on plastic substrate. Etching characteristics are improved because the etching speed does not depend on the crystal orientation in the amorphous phase $[9,10]$. For film bulk acoustic device applications, it is necessary for TC films to be prepared with some specific characteristics. The first one is high resistivity [11], e.g. in an acoustic amplifier semiconductor a resistivity of $10^{3}$ to $10^{6} \Omega \mathrm{cm}$ is required [12]. For touch screens, TC electrodes of sheet resistance in the range of $400-700 \Omega / \mathrm{cm}^{2}$ are required [13].

ITO films have been prepared on polymer substrates using various techniques, such as radio frequency magnetron sputtering [8], ion beam assisted deposition [14], direct current (dc) reactive magnetron sputtering [15], pulse dc magnetron sputtering [16], roll-to-roll dc sputtering [13], dc sputter type negative metal ion source $[17,18]$, etc. To the best of our knowledge the study of ITO prepared by electron beam evaporation technique on polymer substrates has not been reported. Thus, in this contribution, we study the properties of ITO deposited on polymer substrates as a function of ITO film thickness. In particular, we have focused on the electrical resistivity and optical properties at room temperature.

\section{Experimental details}

ITO films were prepared by electron beam evaporation technique from a highly pure $(99.999 \%)$ cold pressed powder of 90 wt. $\% \mathrm{In}_{2} \mathrm{O}_{3}$ and 10 wt. $\% \mathrm{SnO}_{2}$. In order to increase the diffusion process and consequently improve 
the homogeneity of the material, the tablet was heated up to $800^{\circ} \mathrm{C}$ for $3 \mathrm{~h}$. Thin films of the considered ratio was prepared by electron beam evaporation in Edward's high vacuum coating unit model $306 \mathrm{~A}$ under pressure of $5 \times 10^{-6}$ and $9 \times 10^{-5}$ Torr before and during film deposition, respectively. The films were prepared on polymer substrates (Transparency Film CU11-C5, Korea) held at room temperature. The thickness of the films was controlled using digital film thickness monitor model TM 200 Maxtek. The deposition rate was measured to be $\approx 10 \mathrm{~nm} / \mathrm{min}$.

Investigations of the microstructure were carried out using an X-ray diffractometer (Philips model PW1710 X-ray diffractometer). The surface morphology of the ITO films was examined by atomic force microscopy (AFM) using a commercial instrument (Thermomicroscope Co., Autoprobe CP-Research) working in the contact mode. A Jasco V-570 UV-visible-NIR spectrophotometer was employed to record the transmission and reflection spectra over the wavelength $(\lambda)$ range 200$2500 \mathrm{~nm}$ at normal incidence. The optical parameters namely absorption coefficient $(\alpha)$, refractive index $(n)$, and extinction coefficient $(k)$ were calculated as described elsewhere [19]. The sheet resistance $\left(R_{\mathrm{S}}\right)$ of the ITO films were measured with a four-point probe following the procedure proposed by van der Pauw [20].

\section{Results and discussions}

Figure 1a-d shows X-ray diffraction (XRD) patterns of uncoated and ITO coated films with different thicknesses. It is seen from Fig. 1a that the uncoated substrate is characterized by two main crystalline peaks at $2 \theta$ angles $11.93^{\circ}$ and $24.06^{\circ}$. The ITO coatings (Fig. 1b-d) add no additional peaks indicating the amorphous nature for such coatings on the polymer substrate. This finding is in agreement with the work done by You et al. [18] where they prepared ITO on glass and on polyethersulfone (PES) substrates. The ITO/glass films were polycrystalline while the ITO/PES films were amorphous. Hao et al. [15] found that the XRD pattern of the ITO film deposited on polyethylene terephthalate (PET) substrate without pre-deposition surface plasma cleaning showed a typical amorphous amorphous structure. However, all ITO films deposited on PET substrates with predeposition surface plasma cleaning were polycrystalline. This draws the conclusion that nature of the polymer substrate tailors the ITO structure towards amorphous state.

Figure $2 \mathrm{a}-\mathrm{c}$ shows AFM images of the ITO films with different thicknesses. As observed from Fig. 2a, the surface of the $15 \mathrm{~nm}$ film is not uniform and full of hills and craters. As the thickness of the ITO film increased to $50 \mathrm{~nm}$ (Fig. 2b), then $100 \mathrm{~nm}$ (Fig. 2c), the surface uniformity becomes better and the craters disappeared. The uncoated substrate has root mean square (RMS) surface roughness of approximately $2.9 \mathrm{~nm}$. This high roughness of the substrate affects the ITO coated films. The RMS

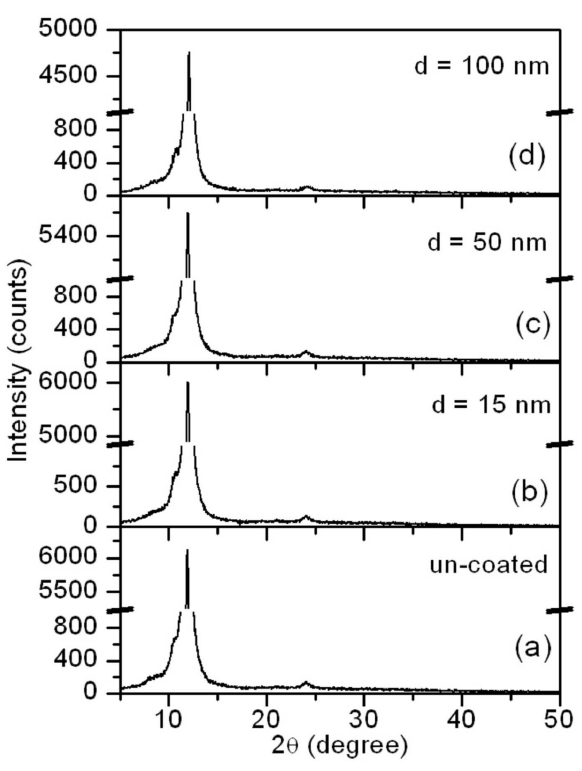

Fig. 1. XRD patterns of ITO thin films deposited on polymer substrates with different thicknesses.

roughness (Fig. 3) increases drastically from $3.2 \mathrm{~nm}$ of the $15 \mathrm{~nm}$ thick film to $7.0 \mathrm{~nm}$ of the $50 \mathrm{~nm}$ thick film, above which the RMS roughness decreases slightly. The stabilization of the surface roughness after certain thickness can possibly ascribed to the filling of the holes and craters, originally started from the bare substrate, by the ITO material.

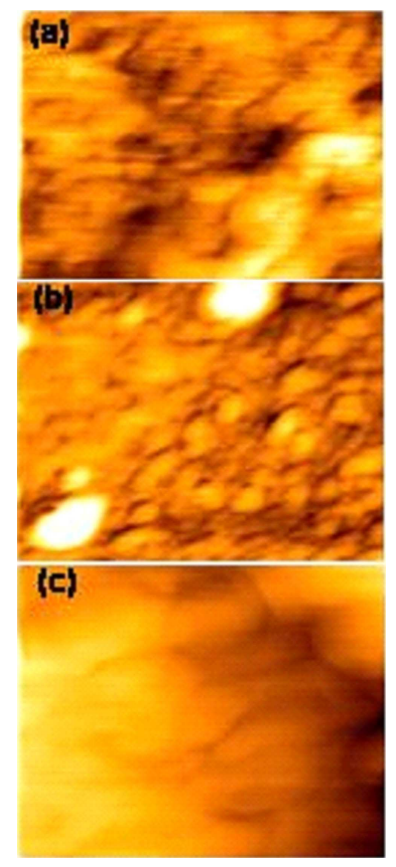

Fig. 2. AFM scans of the ITO films deposited with different thicknesses: (a) $15 \mathrm{~nm}$, (b) $50 \mathrm{~nm}$, and (c) $100 \mathrm{~nm}$. All the scans are $500 \mathrm{~nm} \times 500 \mathrm{~nm}$. The $Z$ axis is $12.5,42$, and $42 \mathrm{~nm}$ for (a), (b) and (c), respectively. 


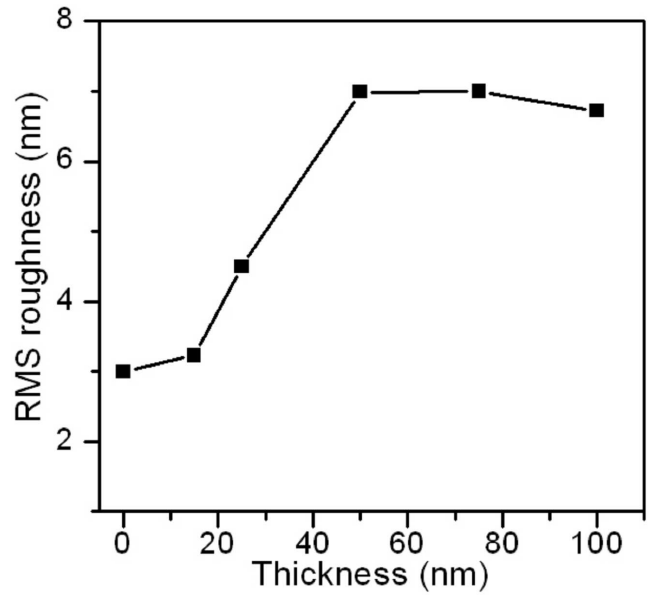

Fig. 3. Variation of the RMS surface roughness with ITO film thickness.

Figure 4 represents the transmittance in the wavelength range from 200 to $2500 \mathrm{~nm}$ for different film thicknesses of ITO thin films in the range of 15-100 nm together with the transmittance of uncoated substrate. The transmittance of the uncoated substrate is very high over the entire visible range and it has four minima (around 1619, 2092, 2290, and $2404 \mathrm{~nm}$ ) in the near infrared range. Apart from the spectra of $15 \mathrm{~nm}$ sample, the transmittances for all the others decrease with increasing film thickness. This is due to the increase in opaqueness, indicating the oxygen vacancies [21], and surface roughness [8]. The absorption edges of the ITO films are not sharp because these films are amorphous and possess impurities due to water vapor adsorbed on the polymer substrate. The minima observed in the transmittance spectra have no corresponding maxima at the reflectivity curves as shown in Fig. 5. Thus it can be positively concluded that these minima are characterizing the absorbing nature of the substrate. Moreover, the reflectivity increases with increasing film thickness.

The optical band gap $\left(E_{\mathrm{g}}\right)$ values calculated from the plot of $(\alpha h \nu)^{2}$ versus $h \nu$ for the ITO films, where $\alpha$ is the absorption coefficient, are shown in Fig. 6. It is observed that $E_{\mathrm{g}}$ decreases from $3.01 \mathrm{eV}$ to $2.90 \mathrm{eV}$ with increasing film thickness. The obtained $E_{\mathrm{g}}$ values are comparable to the values $3.18-2.85 \mathrm{eV}$ for ITO/glass prepared by e-beam evaporation [21]. Figure 7 shows the variations of refractive index in the visible region with the film thickness. It is obvious that the refractive index increases with increasing the film thickness. One can reverse this to the increase in the film density in which the velocity of light in the material would decrease. The extinction coefficient $(k)$ values of ITO films prepared with various thicknesses were determined from $k=\alpha \lambda / 4 \pi$ and are shown in Fig. 8 . The $k$ value decreases with the ITO film thickness up to $75 \mathrm{~nm}$, above which the $k$ value increases progressively with the film thickness. The extinction coefficient is related to the creation of defects and absorption centers.

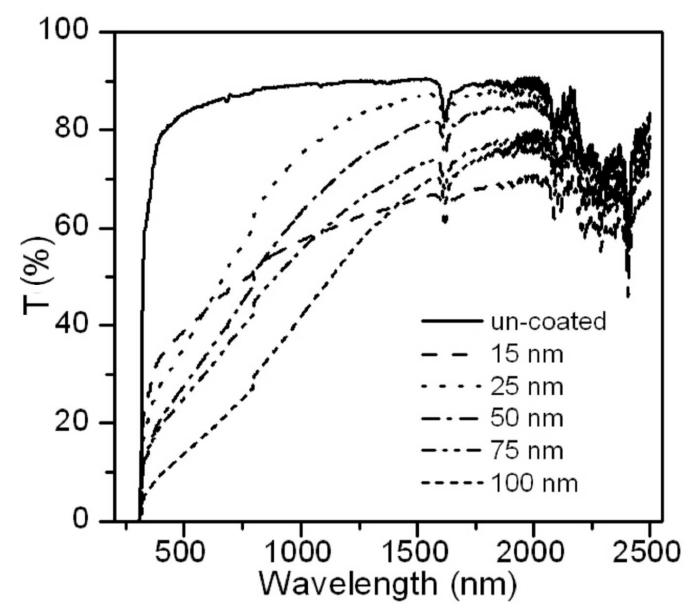

Fig. 4. Spectral transmittance of ITO films as a function of wavelength with different thicknesses.

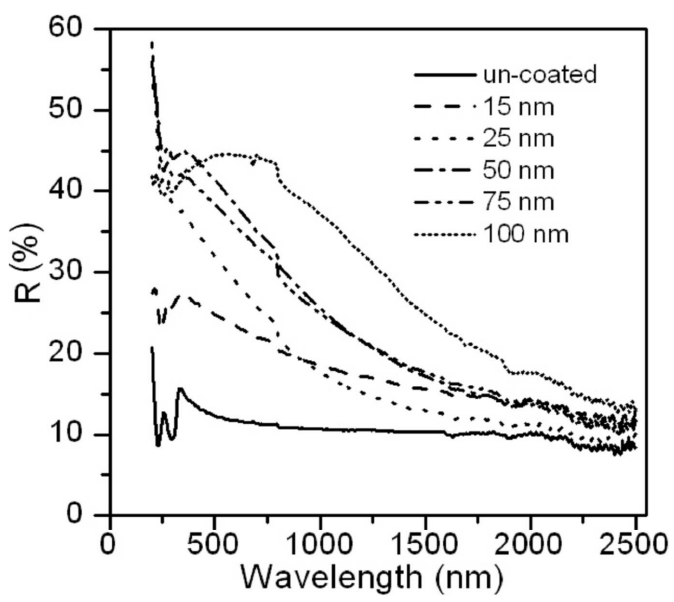

Fig. 5. Spectral reflectance of ITO films as a function of wavelength with different thicknesses.

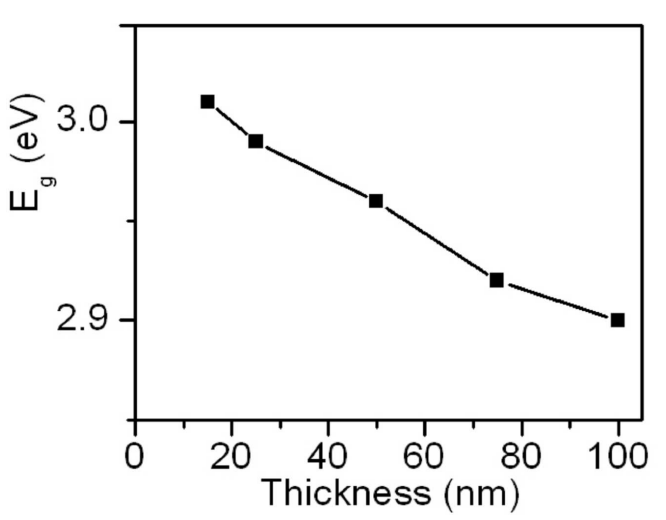

Fig. 6. Variation of the optical band gap with ITO film thickness. 


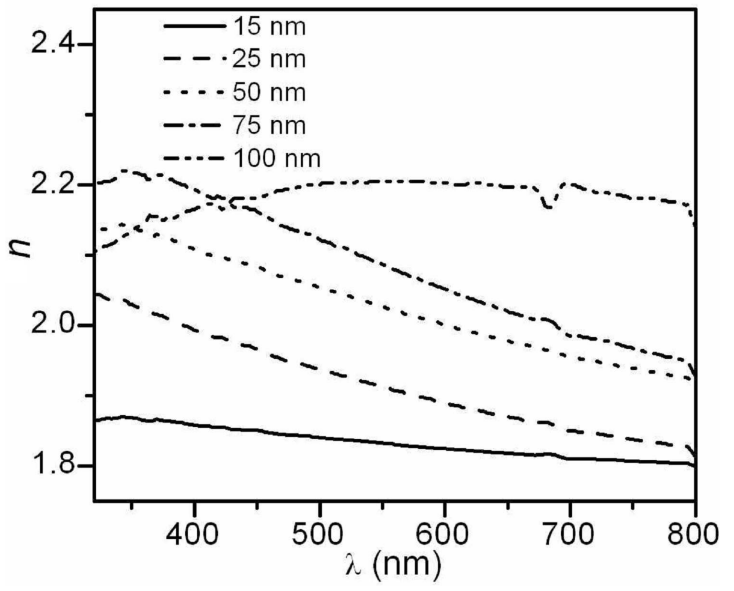

Fig. 7. Refractive index variations of ITO thin films as a function of wavelength deposited with different thicknesses.

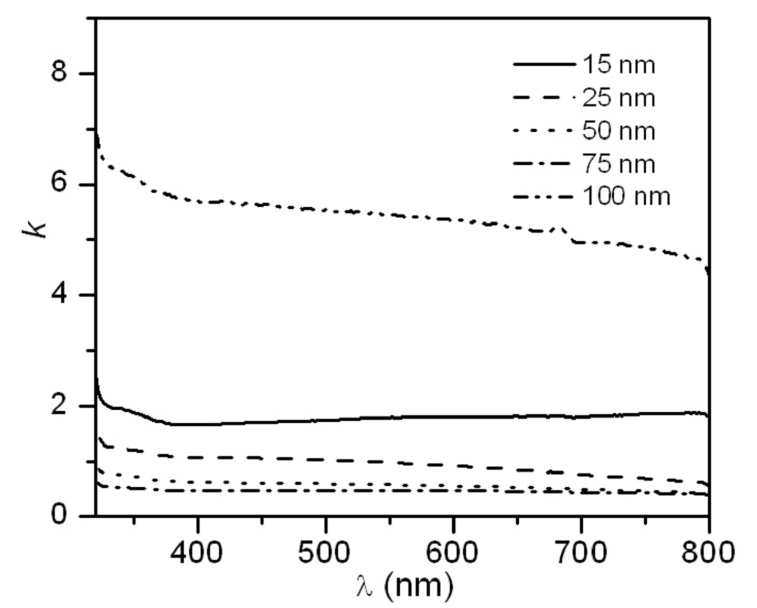

Fig. 8. Extinction coefficient variations of ITO thin films as a function of wavelength deposited with different thicknesses.

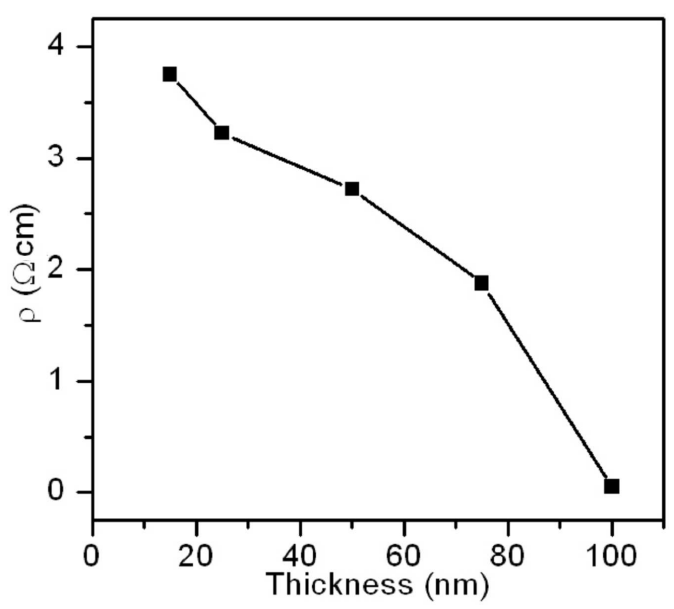

Fig. 9. Dependence of ITO resistivity on film thickness.
Figure 9 shows the variation of the resistivity $(\rho)$ as a function of ITO film thickness. The resistivity was calculated from $\rho=R_{\mathrm{S}} d$. It is observed that the resistivity decreases as the ITO film thickness increases. The obtained resistivity values are higher than the values $7.6 \times 10^{-3}-5.7 \times 10^{-3} \Omega \mathrm{cm}$ obtained by Hao et al. [15] for ITO/PET where the film thicknesses were in the range $72-447 \mathrm{~nm}$. This may be ascribed to the amorphous structure of the obtained films. In amorphous structure films, some electrons are bound in short range by the net non-uniform structure. When the structure of the films becomes crystalline, these electrons are released from the bound. Other factor which can account for the higher resistivity is the possibility of microscopic cracks formation in the ITO layer [22]. Hao et al. [15] stated that in order to get high-quality ITO films of low resistivity, one needs to enhance the crystallinity of the deposited films from the initial stage of deposition and to reduce the thickness of amorphous region on the PET substrate. Several researchers have proposed that the main conduction mechanism of ITO films deposited at low-substrate temperature is due to the creation of oxygen vacancies, which donate two electrons for each vacancy [23-25]. Some of the researchers used plasma surface treatment [26-28] such as oxygen plasma treatment in order to improve ITO conductivity. The plasma surface treatment identified different factors which affected surface electrical properties: (1) removal of surface carbonaceous contamination, (2) changes in the In/Sn ratio indicative of the Sn dopant concentration, (3) changes in the concentration of oxygen vacancies, and (4) producing oxidation of $\mathrm{Sn}-\mathrm{OH}$ surface groups to $\mathrm{Sn}-\mathrm{O}^{*}$ species. Liau et al. [28] proved that the oxidation of $\mathrm{Sn}-\mathrm{OH}$ surface groups to $\mathrm{Sn}-\mathrm{O}^{*}$ species is the most probable factor that enhances ITO conductivity. Thus, presence of $\mathrm{Sn}-\mathrm{OH}$ surface groups on the surface of our ITO films can be accounted as another reason for the obtained high resistivity.

\section{Conclusion}

The effect of thickness of ITO thin films deposited onto polymer substrate by electron beam evaporation technique of a $90 \% \mathrm{In}_{2} \mathrm{O}_{3}-10 \% \mathrm{SnO}_{2}$ cold pressed powder has been studied. It was found that the structural, optical and electrical properties depend strongly on the film thickness. Amorphous structure of all prepared ITO films was found. Moreover, the transmittance, the optical band gap and the resistivity decrease with increasing the film thickness while the refractive index increases. The obtained resistivity values are relatively high but still suitable for the applications as transparent electrode of organic light-emitting diodes, touch screens and in piezoelectric crystal applications.

\section{Acknowledgments}

The authors would like to thank Prof. Dr. M. Wuttig, for his kind permission to carry out the sheet resistance 
at I. Physikalisches Institut1 A der RWTH Aachen, Sommerfeldstr. 14, D-52056 Aachen, Germany. Mr. M. Fathy is acknowledged for his technical support.

\section{References}

[1] I. Hamberg, C.G. Granqvist, J. Appl. Phys. 60, R123 (1986).

[2] K.L. Chopra, S. Major, D.K. Pandya, Thin Solid Films 102, 1 (1983).

[3] C.G. Granqvist, A. Hultaker, Thin Solid Films 411, 1 (2002).

[4] C.M. Lampert, Solar Energy Mater. 6, 1 (1981).

[5] J. Gu, Z. Xiao, M. Xu, G. Wang, Z. Lu, S. Huang, J. Phys. Chem. Solids 59, 27 (1998).

[6] Y.S. Tsai, F.S. Juang, T.H. Yang, M.C. Yokoyama, L.W. Ji, Y.K. Su, J. Phys. Chem. Solids 69, 764 (2008).

[7] M. Gaillet, L. Yan, E. Teboul, Thin Solid Films 516, 170 (2007).

[8] C.-H. Yang, S.-C. Lee, T.-C. Lin, S.-C. Chen, Thin Solid Films 516, 1984 (2008).

[9] K. Tominaga, T. Takao, A. Fukushima, T. Moriga, I. Nakabayashi, Vacuum 66, 505 (2002).

[10] K. Tominaga, H. Fukumoto, K. Kondou, Y. Hayashi, K. Murai, T. Moriga, I. Nakabayashi, Vacuum 74, 683 (2004).

[11] S.-Y. Chu, W. Walter, J.-T. Liaw, J. Eur. Ceram. Soc. 23, 1593 (2003).

[12] E.D. Kolb, R.A. Laudise, J. Am. Ceram. Soc. 49, 302 (1966).

[13] M. Fahland, T. Vogt, W. Schoenberger, N. Schiller, Thin Solid Films 516, 5777 (2008).
[14] L.-J. Meng, J. Gao, M.P. dos Santos, X. Wang, T. Wang, Thin Solid Films 516, 1365 (2008).

[15] L. Hao, X. Diao, H. Xu, B. Gu, T. Wang, Appl. Surf. Sci. 254, 3504 (2008).

[16] Y.C. Lin, J.Y. Li, W.T. Yen, Appl. Surf. Sci. 254, 3262 (2008).

[17] D. Kim, S. Kim, Surf. Coat. Technol. 176, 23 (2003).

[18] Y.Z. You, Y.S. Kim, D.H. Choi, H.S. Jang, J.H. Lee, D. Kim, Mater. Chem. Phys. 107, 444 (2008).

[19] S.H. Mohamed, H.M. Ali, H.A. Mohamed, A.M. Salem, Eur. Phys. J. Appl. Phys. 31, 95 (2005).

[20] L.J. van der Pauw, Philips Res. Rep. 13, 1 (1958).

[21] H.M. Ali, H.A. Mohamed, S.H. Mohamed, Eur. Phys. J. Appl. Phys. 31, 87 (2005).

[22] D.R. Cairns, R.P. Witte II, D.K. Sparacin, S.M. Sachsman, D.C. Paine, G.P. Crawford, R.R. Newton, Appl. Phys. Lett. 76, 1425 (2000).

[23] V. Teixeira, H.N. Cui, L.J. Meng, E. Fortunato, R. Matrins, Thin Solid Films 420-421, 70 (2002).

[24] S. Ohno, Y. Kawaguchi, A. Miyamura, Y. Sato, P.K. Song, M. Yoshikawa, P. Frach, Y. Shigesato, Sci. Technol. Adv. Mater. 7, 56 (2006).

[25] S.H. Shin, J.H. Shin, K.J. Park, T. Ishida, O. Tabata, H.H. Kim, Thin Solid Films 341, 225 (1999).

[26] D.J. Milliron, I.G. Hill, C. Shen, A. Kahn, J. Schwartz, J. Appl. Phys. 87, 572 (2000).

[27] K. Sugiyama, H. Ishii, Y. Ouchi, K. Seki, J. Appl. Phys. 87, 295 (2000).

[28] Y.-H. Liau, N.F. Scherer, K. Rhodes, J. Phys. Chem. B 105, 3282 (2001). 